\title{
Failed Seldinger Technique Deployment of SEMS in Stenosing Mid-Body Stomach Cancer: Overcoming the Problem
}

Yeap SHA*, Tan $\mathrm{CH}$ and Cheng AK

Department of Surgery, Khoo Teck Puat Hospital, Singapore

\begin{abstract}
A case report in which endoscopic attempts at SEMS placement for a stenosing gastric body tumour was not possible using the Seldinger technique, due to difficulty manoeuvring it past an acute angle. A novel technique that was devised to overcome such difficulties is described in this paper.
\end{abstract}

Keywords: Stomach cancer; Dysphagia; Endoscope; Stenosing

\section{Introduction}

Palliative surgical management for gastric carcinoma in its advanced stage or in patients who are poor surgical candidates is often considered to be a high-risk approach with limited effectiveness. There is a high incidence of morbidity and mortality and poor outcomes with persistent symptoms, delayed relief and prolonged hospital stays [1]. Other non-surgical modalities such as balloon dilatation, laser ablation, radiotherapy and feeding tubes only have a transient effect on the symptoms and do not allow adequate oral intake [2].

Self-expandable metal stents (SEMS) have been recognised as an effective, minimally invasive palliative treatment in recent years [3]. Much has been described on endoscopic stenting of the oesophagus, gastric cardia and the gastric outlet. The usual Seldinger method of stent deployment is also well-known. However, the effectiveness of SEMS placement for malignant stenosis of the gastric body is unclear as there have been few reports of such cases, and little literature is available in providing alternative options for difficult stent placements in distorted gastric anatomy. Here, we discuss a case of SEMS placement for a stenosing gastric mid-body tumour which was performed using a novel technique of snaring the stent where the usual Seldinger technique failed. The usefulness, safety and problems of this method and treatment are discussed.

\section{Case Presentation}

An 86-year old man of Malay ethnicity with a history of congestive cardiac failure with poor systolic function was referred from the community clinic to our hospital's gastroenterologist for complaints of 1-month duration of dysphagia, burping and intermittent vomiting which was associated with significant loss of appetite and weight. Endoscopic evaluation revealed a $10-\mathrm{cm}$ long circumferential stricture of the mid-body of the stomach with nodular and friable mucosa (Figure 1) with the presence of a large food bolus retained proximal to the stricture. Biopsy specimens obtained from that region showed features of an ulcerated, signet ring cell variant of poorly differentiated mucin-secreting adenocarcinoma. A computed tomography scan of his thorax, abdomen and pelvis revealed no lesions suspicious for metastases. Options of surgery (including a curative resection or a gastro-jejunal bypass), SEMS placement and permanent naso-jejunal feeding were offered and discussed with both the patient and his family. In view of his advanced age and significant cardiac comorbidity, he was deemed to be a high-risk candidate for prolonged general anaesthesia and major surgery. After discussion with the patient and his family, the decision was finally made for SEMS placement to achieve relief of symptoms and improve his perceived quality of life. The procedure would still be performed under general anaesthesia for airway protection.

The patient was placed in supine position. A guide-wire was initially placed with endoscopic guidance and the proximal and distal extents of the stenosis were marked with artery forceps under radiological guidance. A Boston Scientific Wallflex esophageal partially covered 23 $\mathrm{mm} \times 125 \mathrm{~mm}$ stent was selected. Multiple attempts made to insert the SEMS over the pre-placed guide-wire were hampered by increased rigidity of the stomach caused by the mid-body tumour, as well as distorted anatomy causing an acute angle between the cardia and the mid-body (Figure 2). Deployment of the guidewire in the usual fashion led to kinking of the delivery device as it was unable to follow the wire. Further attempts in using various endoscopic graspers (such as a biopsy grasper and a rat-tooth grasper) to navigate the SEMS over the guide-wire failed as well. We then anchored the SEMS to the tip of the gastroscope using a snare (Figure 3). This allowed the gastroscope to guide the SEMS across the acute angle and pass it beyond the distal end of the stenosis under direct vision without a guide-wire. After the stent was released from the snare, it was then slowly deployed by removing the outer catheter and pulling back the endoscope. Successful stent placement was confirmed with intraoperative fluoroscopy (Figure 4).

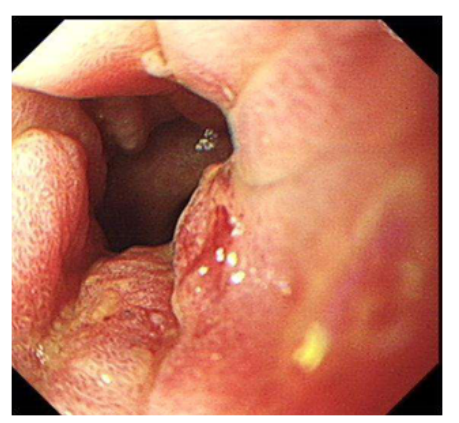

Figure 1: Endoscopic view of circumferential structure at mid-body.

*Corresponding author: Yeap SHA, 120 Tampines, Street 11 \#12-296, Singapore Tel: 6582826321; E-mail: amelia.yeap@mohh.com.sg

Received March 30, 2016; Accepted April 11, 2016; Published April 21, 2016

Citation: Yeap SHA, Tan CH, Cheng AK (2016) Failed Seldinger Technique Deployment of SEMS in Stenosing Mid-Body Stomach Cancer: Overcoming the Problem. Med Rep Case Stud 1: 110. doi: 10.4172/2572-5130.1000110

Copyright: (C) 2016 Norinsky AB, et al. This is an open-access article distributed under the terms of the Creative Commons Attribution License, which permits unrestricted use, distribution, and reproduction in any medium, provided the original author and source are credited. 


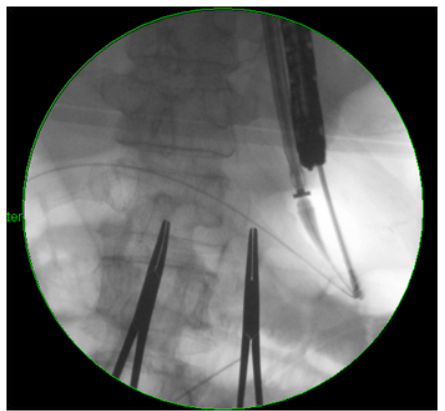

Figure 2: Acute angle between the cardia and the mid-body, with artery forceps marking proximal and distal ends of the stenosis.

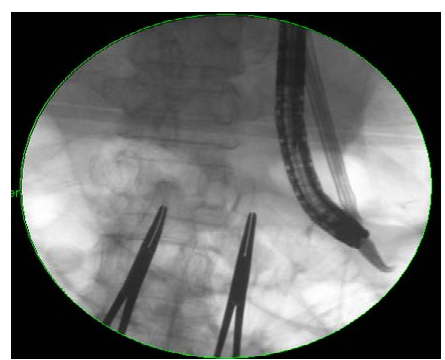

Figure 3: SEMS anchored to tip of gastroscope using snare.

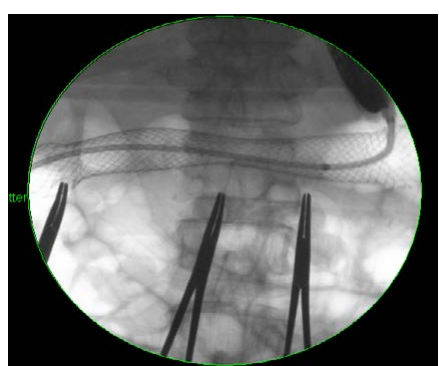

Figure 4: Successful stent placement with waisting seen.

No adverse events were observed during the SEMS placement, and our patient recovered well post-procedure. He tolerated a gradual escalation of feeding regime, and was discharged on post-operative day 2. He was subsequently referred to Palliative Medicine for further care, and a repeat X-ray on post-operative day 8 showed no stent migration.

\section{Discussion}

Stent placement of the oesophagus, gastric outlet and duodenum has been well documented, but few reports of stenting for gastric body carcinomas were found. This may be due to the diffuse nature of tumours that present in this area. Thus, appropriate selection of candidates is crucial in ensuring the success of stent placement in gastric body tumours - those with a more localised short segment stenosis may be more amenable to stent placement, while those with a longer segment would tend to encounter more difficulties. Possible problems with stenting gastric body tumours could include a higher risk of stent migration. This may be mitigated by the use of uncovered stents which potentially increases friction forces between the stent and the gastric mucosa [4].

Methods of stent placement that have been commonly described include wire guidance, as in the Seldinger technique, and through the scope delivery, often with the help of fluoroscopy. Failures of stent placement, when encountered, have been reported as due to failure to gain access to the obstruction due to complicated anatomy, severe obstruction, and stent positioning and deployment issues [5]. A recent case report has described the author's use of a balloon overtube-assisted approach for difficult stent placement [6], but little current literature has described in detail any other methods used to overcome technical difficulties in stent deployment.

This case report attempts to fill the gap in literature by providing an additional option in cases of failed stent deployment using the Seldinger technique. This method of inserting both stent and scope anchored with a snare, as described in our patient, has several advantages. It improves the endoscopist's ability to manoeuvre the stent and better negotiate difficult or distorted anatomy without additional need for costly devices. It also allows safe deployment under simultaneous endoscopic and fluoroscopic visualization. Endoscopic snares are readily available, affordable and are able to secure the delivery device to the endoscope. It is a safe method and would have a role in ameliorating problems encountered with stent deployment.

Further study of the various technical modifications used in endoscopic stent placements and their outcomes may be worthwhile in contributing to advances in the field and innovative engineering of future delivery devices.

\section{Conclusion}

The above case is one of very few reported successful stent placements for stenosing gastric mid-body tumor in current literature. Stent placement for these tumours is a feasible management option and should be actively considered in the palliative management of a selected group of patients. The technique of "snare over stent" can be used to successfully to negotiate past difficult angles during stent placement and should be considered in challenging cases of stent navigation. Other methods of using a biopsy grasper or a rat-tooth grasper may also be effective.

\section{References}

1. Dormann A, Meisner S, Verin N, Wenk Lang A (2004) Self-expanding metal stents for gastroduodenal malignancies: Systematic review of their clinical effectiveness. Endoscopy 36: 543-550.

2. Tendler DA (2002) Malignant gastric outlet obstruction: Bridging another divide Am J Gastroenterol 97: 4-6.

3. Lye TJ, Goh YC, Eng AK, Ong HS, Wong WK et al. (2014) Endoscopic self-expandable metallic stenting for palliation of malignancy gastric outlet obstruction in Southeast Asia. ANZ J Surg.

4. Pan YM, Pan J, Guo LK, Qiu M, Zhang JJ, et al. (2014) Covered versus uncovered self-expandable metallic stents for palliation of malignant gastric outlet obstruction: A systematic review and meta-analysis. BMC Gastroenterol 14: 170

5. Baron TH, Schöfl R, Puespoek A, Sakai Y (2001) Expandable metal stent placement for gastric outlet obstruction. Endoscopy 33: 623-628.

6. Neumann H, Wilcox CM, Mönkemüller K (2013) Balloon overtube-assisted placement of self-expanding metal stents. Endoscopy 45: E369-E370. 